\title{
INTERVENÇÃO DIDÁTICA NA EDUCAÇÃO DE JOVENS E ADULTOS: ABORDAGEM INTERDISCIPLINAR ACERCA DAS DOENÇAS CRÔNICAS NÃO TRANSMISSÍVEIS
}

\author{
Laís Tamiris das Neves Felizardo da Luz ${ }^{1}$ e Gabriela Rebelo da Silva ${ }^{2}$ \\ ${ }^{1}$ Graduada em Ciências da Natureza com habilitação em Química pelo Instituto Federal de \\ Santa Catarina (Ifsc) e Pós-graduada em Educação de Jovens e Adultos pela Faculdade \\ São Luís. \\ ${ }^{1}$ Bacharel e Licenciada em Educação Física pela Universidade do Estado de Santa Catarina \\ (Udesc) e Pós-Graduada em Gestão e Metodologia do Ensino Interdisciplinar pela \\ Faculdade Dom Bosco. \\ lais_tamiris@hotmail.com, bielars4@yahoo.com.br \\ Acesso DOI: http://dx.doi.org/10.34059/ciejop.2019v28i1-5
}

\section{Resumo}

FELIZARDO DA LUZ, L.T; REBELO DA SILVA, G. Intervenção didática na educação de jovens e adultos: abordagem interdisciplinar acerca das doenças crônicas não transmissíveis. Revista Científica JOPEF, Vol.28, n.1, pp.54-61, 2019. Neste artigo, discutimos uma intervenção didática acerca da temática Doenças Crônicas Não Transmissíveis ligadas aos hábitos alimentares e de vida. A ação foi desenvolvida com três turmas de $2^{\circ}$ ano do Ensino Médio da Educação de Jovens e Adultos (EJA) nas disciplinas de Educação Física e Química. Por estratégia, utilizamos, primeiramente, termos presentes no dia a dia dos alunos e, a posteriori, a substituição para linguagem adequada cientificamente, visando melhor compreensão da temática por parte dos alunos. Salientamos, com base nos resultados, a importância de intervenções interdisciplinares que promovam melhorias no estilo de vida e que busquem impulsionar o interesse dos alunos nessas áreas através de curiosidades acerca do funcionamento do nosso corpo e da relação dele com os alimentos e as atividades físicas. Outrossim, buscamos aproximar os sujeitos da área da química, por meio da explanação dos tipos de reações químicas que ocorrem em nosso corpo, de acordo com a dieta e o estilo de vida que seguimos. Após desenvolvimento da ID, a compreensão dos alunos foi aperfeiçoada. Assimilaram com facilidade o quanto a alimentação adequada e bons hábitos podem influenciar positivamente na qualidade de vida e mostraram-se dispostos a mudanças.

Palavras-chave: Doenças Crônicas. Interdisciplinaridade. Intervenção didática.

\section{TEACHING INTERVENTION IN YOUTH AND ADULT EDUCATION: INTERDISCIPLINARY APPROACH ABOUT NON-TRANSMISSIBLE CHRONIC DISEASES}

FELIZARDO DA LUZ, L.T; REBELO DA SILVA, G. Teaching intervention in youth and adult education: interdisciplinary approach about non-transmissible chronic diseases. Revista Científica JOPEF, Vol.28, n.1, pp.54-61, 2019. In this article, we discuss a didactic intervention on the theme Noncommunicable Chronic Diseases linked to eating and life habits. The action was developed with three groups of 2nd year of High School of Youth and Adult Education (EJA) in the subjects of Physical Education and Chemistry. For strategy, we use, firstly, terms present in the students' daily life and, subsequently, the substitution for scientifically adequate language, aiming at a better understanding of the subject by the students. Based on the results, we stress the importance of interdisciplinary interventions that promote lifestyle improvements and seek to stimulate students' interest in these areas through curiosities about the functioning of our body and its relationship with food and physical activities. Furthermore, we seek to bring the subjects closer to the area of chemistry, by explaining the types of chemical reactions that occur in our body, according to the diet and lifestyle we follow. After the development of ID, students' understanding was improved.

Fórum Internacional de Qualidade de Vida e Saúde - Balneário Camboriú/SC, 12 de outubro de 2019 
They easily assimilated how well eating and good habits can positively influence quality of life and were willing to change.

Keywords: Chronic Diseases. Interdisciplinarity. Didactic intervent.

\section{RELATO DE EXPERIÊNCIA}

O presente trabalho resulta de um relato de experiência desenvolvido em um Centro de Educação de Jovens e Adultos (CEJA) de Tijucas/SC, cuja proposta inicial foi abordar com os alunos do $2^{\circ}$ ano do Ensino Médio, na disciplina de Educação Física e Química, a importância de adotarmos bons hábitos de vida. Buscamos conscientizar os alunos acerca da manutenção da saúde por meio de alimentos adequados e variados - a fim de sanar deficiências nutricionais e prevenir doenças infecciosas. Destacamos a importância da regularidade da hidratação corporal e da prática da atividade física para alcançar tais objetivos.

Para abordar a temática proposta de modo contextualizado, buscamos a relação da mesma com a alimentação e o estilo de vida moderno, vinculando-os aos conhecimentos da área da Educação Física e da Química.

A Intervenção Didática ${ }^{1}$ (ID) não teve apenas por objetivo contextualizar as aulas de Educação Física e Química a partir da realidade vivenciada pelos alunos, mas também aproximá-los da área de Ciências da Natureza e instigá-los a buscar melhor condicionamento físico, adotando um estilo de vida saudável, superando o sedentarismo.

A seguir, apresentamos a metodologia utilizada nesta ID, seguida da discussão dos resultados e da conclusão.

\section{O CONTEXTO DA TEMÁTICA NO COTIDIANO DO CEJA}

O CEJA é composto por alunos na faixa etária entre quinze e sessenta anos de idade. Frente a isso, destacamos que, em uma sala, há diversidade de conhecimentos e dificuldades por partes dos educandos. Com isso, buscamos ensinar da forma mais didática, lúdica e contextual possível, para proporcionar aos

\footnotetext{
${ }^{1}$ A Intervenção didática (ID) objetiva, por meio de uma ação-intervenção, a possibilidade de "tomada de decisão" para gerar mudanças. Espera-se que ocorram oportunidades para que mudanças mobilizadas por uma ação consciente aconteça. Uma ID deve ter como ponto de partida a realidade dos estudantes, e, ao ser concluída, deve oferecer algo para a transformação dessa (Zwierewicz, 2014).
}

Fórum Internacional de Qualidade de Vida e Saúde - Balneário Camboriú/SC, 12 de outubro de 2019 
alunos ensino de qualidade e instigá-los ao conhecimento, uma vez que muitos deles estão há mais de vinte anos afastados da escola.

Assim sendo, inicialmente, adotamos o uso da linguagem coloquial para explicar e exemplificar o conteúdo referente à temática, com o intuito de aproximar e envolver os alunos na ID, uma vez que percebemos que, desse modo a compreensão dos alunos acerca de determinado fenômeno, modelo, teoria, conceito e/ou lei estudado se dá de forma mais harmônica e prazerosa.

Em contrapartida, causa-nos uma certa preocupação o modo como as indústrias alimentícias estabelecem a comunicação entre produto-consumidor, uma vez que fazem uso de uma linguagem técnico-científico que dificulta a compreensão de informações relevantes por parte da maioria dos consumidores. A dificuldade em interpretar corretamente informações dos rótulos pode contribuir para a ingestão de alimentos ultraprocessados. Estes são a base da má alimentação, o que influencia negativamente o desenvolvimento das Doenças Crônicas Não Transmissíveis (DCNT).

Grande parte dos consumidores não leem as informações nutricionais dos alimentos antes de consumi-los. Para Bendino (2012, p.4), "os consumidores têm dificuldade em relação ao entendimento e uso das informações dos rótulos e da informação nutricional para a manutenção da saúde".

A má alimentação da sociedade atual, assim como o sedentarismo, é fator que vem contribuindo para o desenvolvimento das DCNT, como hipertensão arterial, enfermidades cardiovasculares, acidentes cerebrovasculares, infarto, câncer, diabetes e doenças respiratórias crônicas, entre outras (Buzzo, 2014 \& Brasil, 2014). Atualmente, as DCNT têm se tornado um agravante problema na saúde pública. Conforme Goulart (2011, p.14),

O consumo de alimentos com altas taxas de gorduras saturadas e trans, de sal e de açúcar é a causa de ao menos 14 milhões de mortes ou de $40 \%$ de todas as mortes anuais por DCNT e estas, representam hoje a maior causa de morte no mundo, sendo que em 2008 foram 36 milhões de mortes, ou seja, $63 \%$ do total global de mortes (Goulart, 2011, p.14 grifo nosso).

Essas doenças multifatoriais desenvolvem-se no decorrer da vida e são de longa duração. Elas são resultado de diversos fatores, determinantes sociais e condicionantes, além de fatores de risco individuais, como tabagismo, consumo excessivo de álcool, inatividade física e alimentação não saudável (Brasil, 2014). 
A Organização Mundial da Saúde (OMS), devido ao crescente e alarmante casos de DCNT, criou a Estratégia Global para Promoção da Alimentação Saudável, Atividade Física e Saúde e propôs parceria com a Iniciativa Escolas Amigas da Nutrição (IEAN), que visa incluir temas relacionados a nutrição alimentar nas escolas, como, por exemplo, o Guia Alimentar para a População Brasileira (GAPB), que tem por intuito substituir a pirâmide alimentar e auxiliar educadores e toda comunidade a adotar hábitos nutricionais e de vida saudáveis.

De acordo com a OMS (2015), a maioria das mortes prematuras (antes dos 70 anos) por DCNT são evitáveis. No entanto, faz-se necessário incentivar a comunidade escolar, assim como toda a população, a priorizar os alimentos in natura e evitar os ultraprocessados, além de praticar atividade física diariamente, como propusemos aos nossos alunos.

Figura 1 - Alongamento: preparação para os exercícios físicos

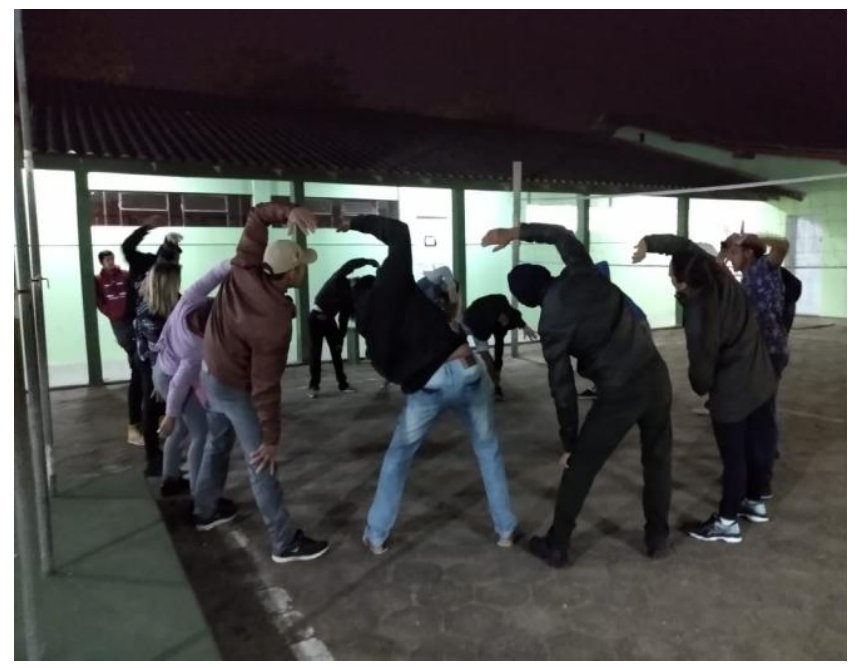

FONTE: Das autoras

De acordo com a Agência Nacional de Vigilância Sanitária (Anvisa), "A escolha de alimentos saudáveis reduz o risco de certas doenças, como obesidade, diabetes, câncer e hipertensão" (Brasil, 2001a, p.7).

A alimentação, quando adequada e variada, pode prevenir deficiências nutricionais e proteger contra doenças infecciosas, porque é rica em nutrientes os quais melhoram as defesas do organismo. Em contrapartida, os alimentos industrializados conferem agilidade e praticidade ao nosso dia a dia, mas comprometem a nossa saúde.

Por isso, é indispensável uma alimentação e estilo de vida saudável, a ingestão, de forma variada, de alimentos naturais, dando preferência aos integrais e 
orgânicos e excluindo do cardápio os produtos ultraprocessados, como os refrigerantes, fast food, biscoito recheado, caldos e temperos prontos, margarina e outros, que, além de não serem nutritivos, podem prejudicar nosso organismo (Carmo, 2006).

\section{CAMINHOS DA PESQUISA}

Iniciamos a ID instigando os alunos por meio de perguntas acerca da temática “Doenças Crônicas Não Transmissíveis ligadas aos hábitos alimentares e de vida", com intuito de identificar os conhecimentos prévios deles acerca da mesma.

Posteriormente, trabalhamos com esses conhecimentos prévios, relacionandoos com alguns conceitos sobre atividade física e saúde, tais como: [i] qualidade de vida; [ii] alimentação; [iii] hidratação; [iv] estilo de vida; [v] sedentarismo; [vi] doenças; [vii] alongamentos; [viii] caminhada; [ix] visitas a academias públicas; [x] esportes; [xi] dança, entre outros.

Figura 2 - Dança: expressão/linguagem corporal

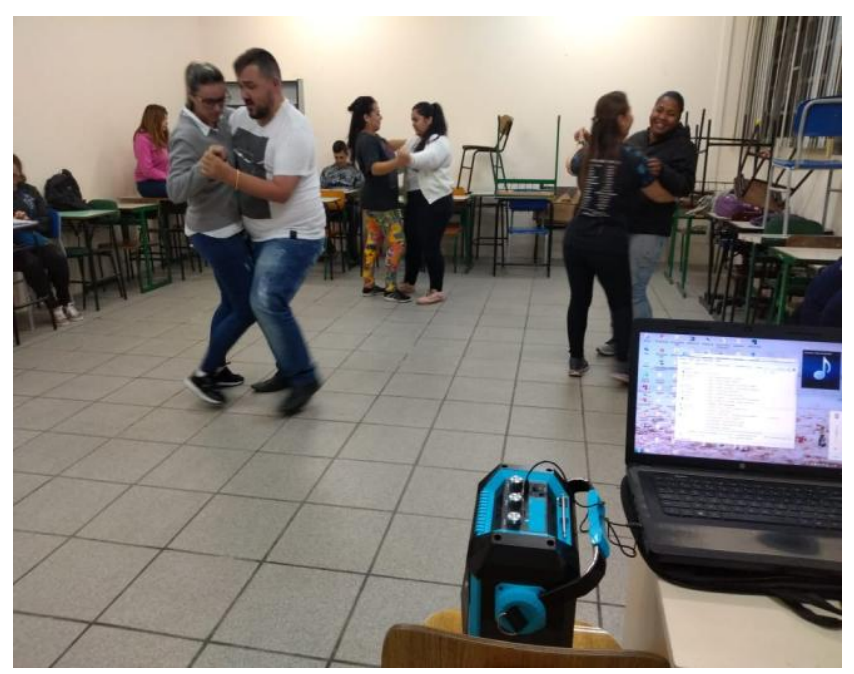

FONTE: Das autoras

Do mesmo modo, trabalhamos com conceitos químicos referentes à temática, tais como: [i] concentração comum; [ii] radicais livres; [iii] ligações químicas; [iv] anabolismo e catabolismo; [v] elementos químicos; [vi] energia contida nos alimentos; [vii] doença crônicas; [viii] classificação dos alimentos, entre outros. 
Figura 3 - Lúdico: jogo didático acerca das classificações dos alimentos

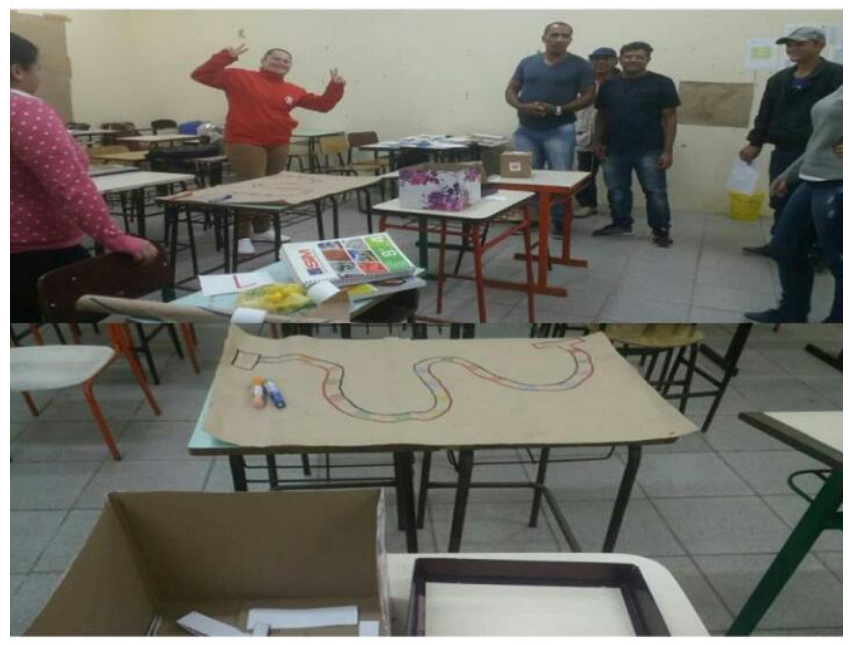

FONTE: Das autoras

A ID foi realizada em três turmas de $2^{\circ}$ ano do Ensino Médio. Para a realização desta ID, foram utilizadas três semanas de aulas, totalizando quinze aulas - cinco aulas faixa de 45 minutos por semana para cada uma das turmas.

Entendemos que exemplos contextualizados e ligados ao cotidiano como os mencionados acima são uma forma de possibilitar um aprendizado mais satisfatório, que leva os alunos a refletirem e a tornarem-se mais críticos.

Ao término das atividades, os alunos foram instruídos a produzirem um relato, no qual deveria conter seus conhecimentos prévios e os adquiridos após a ID, assim como suas curiosidades e inquietações a respeito da temática em questão. Para tal, refizemos os mesmos questionamentos que havíamos realizado no início desta ID. A seguir, apresentamos uma breve discussão em torno dos relatos dos alunos.

\section{RESULTADOS E DISCUSSÕES}

A partir da realização da ID descrita neste relato, os alunos foram capazes de apropriar-se a respeito de temas ligados a nutrição em ambas as disciplinas. Foi perceptível o envolvimento dos alunos no decorrer das aulas, uma vez que a temática em questão despertou curiosidade e interesse e os fez relacionar com situações do cotidiano, ampliando seus conhecimentos.

Percebemos que tal compreensão deu-se pelos exemplos apresentados, pois esses estavam vinculados ao dia a dia da turma e também pelo uso da linguagem coloquial em conjunto com a linguagem científica. 
Assim, percebemos que, após a ID, a compreensão dos alunos foi aperfeiçoada. Ao serem questionados sobre o que eles concluíram, os alunos responderam que agora percebem mais facilmente o quanto uma alimentação adequada e bons hábitos podem influenciar na qualidade de vida.

Após a ID, alguns alunos expuseram suas impressões e conclusões sobre a temática e enunciaram comentários do tipo: "vou procurar me cuidar mais quanto à alimentação"; "tenho que começar a fazer exercícios"; "sou sedentário de carteirinha, tenho que começar a me cuidar"; "na minha família, quase todos têm problemas de hipertensão e colesterol"; "a química está presente em nosso dia a dia"; "é necessário ter uma alimentação saudável para equilibramos os metais que temos dentro do nosso corpo"; "devemos substituir as panelas de alumínio"; "preciso melhorar meu estilo de vida, vou começar mudando a minha alimentação e praticar alguma atividade física", entre outras considerações.

Como troca mútua de conhecimentos, esta ID oportunizou rever conceitos químicos e refletir sobre a prática docente. Muitas estratégias utilizadas - ilustrações no quadro branco, exemplos ligados ao cotidiano, desenhos, uso da linguagem coloquial juntamente com a científica - permitiram compreender o modo como os alunos desenvolvem conhecimentos em Química e em Saúde.

\section{CONCLUSÃO}

Concluímos que o tema abordado foi relevante, uma vez que possibilitou abordarmos conceitos referentes à Educação Física e à Química, além de hábitos alimentares, hábitos de vida e a origem de algumas doenças ligadas diretamente ao estilo de vida - má alimentação e sedentarismo - como as DCNT.

Destacamos a importância do planejamento das estratégias didáticas, pois com ideias bem organizadas, objetivos bem estabelecidos e buscando fazer relações entre conteúdos científicos e a vivência dos alunos, é possível propiciar condições que possibilitem a obtenção de êxito na atividade realizada, favorecendo a aprendizagem.

Por fim, concluímos que a ID, quando planejada com temas que remetem ao cotidiano e interesse dos alunos e que faça uso da linguagem coloquial seguida da técnica contribui favoravelmente para o aprendizado e aproxima os alunos das áreas das Ciências, uma vez que esses passam a vê-la com maior naturalidade e afinidade.

Fórum Internacional de Qualidade de Vida e Saúde - Balneário Camboriú/SC, 12 de outubro de 2019 


\section{REFERÊNCIAS}

BENDINO, N. I; et al. Avaliação do conhecimento e dificuldades de consumidores frequentadores de supermercado convencional em relação à rotulagem de alimentos e informação nutricional. Journal of the Health Sciences Institute, v. 30, n. 3, p. 261-265, 2012.

BRASIL. Ministério da Saúde. Agência Nacional de Vigilância Sanitária. Rotulagem nutricional obrigatória: manual de orientação aos consumidores, educação para o consumo saudável. Brasília, DF: Ministério da Saúde, 2001. Disponível em:< http://www.anvisa.gov.br/alimentos/rotulos/manual_rotulagem.pdf>. Acesso em:08 jul. 2019.

BRASIL. Ministério da Saúde. Portal da Saúde - Vigilância das Doenças Crônicas Não Transmissíveis, 2014. Disponível em: http://portalsaude.saude.gov.br/index.php/o-ministerio/principal/leia-mais-oministerio/671-secretaria-svs/vigilancia-de-a-a-z/doencas-cronicas-naotransmissiveis/14125-vigilancia-dasdoencas-cronicas-nao-transmissiveis>. em: 20 ago 2019.

BUZZO, M. L. et al. Elevados teores de sódio em alimentos industrializados consumidos pela população brasileira. Revista do Instituto Adolfo Lutz, v. 73, n. 1, p. 32-39, 2014.

CARMO MB. TORAL N. SILVA MV. SLATER B. Consumo de doces, refrigerantes e bebidas com adição de açúcar entre adolescentes da rede pública de ensino de Piracicaba, São Paulo. Rev Bras de Epidemiol. 2006.

GOULART. F. A. A. Doenças crônicas não transmissíveis: estratégias de controle e desafios para os sistemas de saúde. Brasília: Organização panamericana da saúde, 2011.

OMS. Organização Mundial de Saúde. Determinantes Sociais e Riscos para a Saúde, Doenças Crônicas não transmissíveis e Saúde Mental, 2015. Disponível em: $<$ https://www.paho.org/bra/index.php?option=com_content\&view=article \&id=4766:do encas-cronicas-nao-transmissiveis-causam-16-milhoes-de-mortes-prematuras-todosos-anos\&ltemid=839>. Acesso em: 18. ago. 2019.

ZWIEREWICZ M. Material didático curso de Pós-Graduação Especialização Educação Profissional Integrada à Educação Básica na Modalidade de Educação de Jovens e Adultos - PROEJA. Ủnidade Curricular SEMINÁRIO DE PESQÜISA E INTERVENÇÃO I. Florianópolis -SC. IFSC, 2014. 